\section{Opiate overdose in Dublin}

Dear Editor,

Patients who overdose on opiate drugs have a huge impact on A\&E departments due to the workload they generate and their associated mortality. ${ }^{1}$ A prospective audit of all cases presenting with clinical evidence of opiate overdose was performed over a six-month period which coincided with the introduction of new methadone prescribing legislation ${ }^{\wedge}$

One hundred and twenty-eight cases were identified. The mean age was 26 years and $73 \%$ were male. Forty-four per cent had taken other drugs. $2 \%$ presented on more than one occasion and $1 \%$ died during the episode in question. These statistics were all considerably less than those reported in other studies.'

Despite having critical physiological characteristics and often requiring emergency treatment, $65 \%$ discharged themselves against medical advice. A $32 \%$ increase in victims of acute opiate overdose was observed in the two-month period immediately after the introduction of the new methadone prescribing legislation, with a subsequent drop (47\%) two months later $\left(x^{2}:=8.0, p<0.05\right)$.

Although victims of acute opiate overdose are often critically ill, providing definitive treatment can be difficult and A\&E departments have an important role to play in monitoring patterns of opiate drug use.

\section{W Cullen ${ }^{1}$, S Griffin ${ }^{2}$, G Bury ${ }^{1}$, PK Plunkett ${ }^{2}$}

Department of General Practice', University College Dublin, Department of Emergency Medicine ${ }^{2}$, St James's Hospital, Dublin, Ireland.

\section{References}

1. Desmond N, Murphy M, Plunkett P, Mulcahy F. Use of a Dublin inner city A\&E department by patients with known HIV-1 infection, hit f STD AIDS 1993; 4: 222-5.

2. Misuse of drugs (supervision of prescription and supply of methadone) regulations (Dublin, Stationery Office, Statutory Instrument 225): Department of Health and Children, 1998.

3. Seidler D, Stuhlinger G, Fischer Ci et al. After antagonisation of acute opiate overdose: a survey at hospitals in Vienna. Addiction 1996; 91: 1479-87.

\section{Cannabis abuse in Iran}

\section{Dear Editor,}

Virtually every aspect of public life in Iran was affected by the Islamic revolution in 1979. Many government policies were altered having been considered unIslamic, including the drug policy. The new regime made alcohol a prime target and provided a new national campaign against drug abuse. Drug laws became stricter with extensive use of the death penalty for drug trafficking. The war with Iraq affected the economy and social order, including the drug programme. We have only official press announcements on the extent and success of the drug campaign.

Little is known about substance dependence in Iran. The penalty for possession and use of all illegal substances is arrest and probable imprisonment. Alcohol is both religiously and legally prohibited. Illicit substances are cannabis, heroin, opium, alcohol, stimulants, LSD and hallucinogens. About $60 \%$ of the Iranian population are younger than 25 years of age, a large proportion of these are students.

We sampled 1,500 subjects aged 15 years and older, in
Shiraz, the capital of the Fars Province (population 1.5 million). Subjects were chosen by area and cluster random sampling. Shiraz was divided into 15 geographic areas and each cluster included 100 subjects. Studies were conducted using a structured interview based on DSM-IV and ICD-10 criteria for cannabis abuse and dependence. A short explanation of the study and $A n$ assurance of confidentiality were given. Interviews were conducted in the subjects' homes. There were questions on age, gender, marital status and past and present cannabis use, and reasons for cannabis use. Data was analysed usint ${ }^{\wedge}$ SPSS programme. A X" test tested frequencies and a t-test was used to compare means.

The overall response rate was $93 \%$, with 1,400 participants. One hundred subjects were excluded due to incomplete data. Fifty per cent of participants were men. The mean age for men was 35 years i SD 14.06; range 15-80) and women was 31 years (SD 12.90; range 15-83) $(\mathrm{p}<0.001)$. The majority $(36 \%)$ were 15-24 years of age, $27 \%$ were $25-34$ and $18 \%$ were $35-44$. A total of $88(6.3 \%)$ admitted using cannabis once or more during their lives -80 of whom were men, 17 were cannabis abusers (all men) and 18 were cannabis dependent (13 men). The most frequent reason for initial cannabis use was curiosity and for current use seeking pleasure. Users were most frequently self-employed, followed by government employees and university students $(\mathrm{p}<0.05)$. Use was greatest amongst the well educated followed by diploma holders, high school students and illiterates $(\mathrm{p}<0.05)$.

Cannabis use was greater in males, in contrast with Western studies showing no gender difference.' In Iran, women are socially downgraded if they smoke or drink. This research was confined to Shiraz, so care must be taken not to generalise these findings to the entire population.

\section{J Ahmadi, M Sharif!}

Department of Psychiatry, PO Box 71345-1416, Hafez Hospital, Shiraz University of Medical Sciences, Shiraz, Iran. Email: jamshid_ahmadi@yahoo.com

\section{Reference}

1. Gulliver SB, Kalman D, Rohscnow DJ. Smoking and drinking among alcoholics in treatment: cross-sectional and longitudinal relationships. / Alcohol 2000; 61 (1): 157-63.

\section{Prostatic carcinoma presenting as testicular mass}

Dear Editor,

Metastases to the testis are rare but when present the prostate should be considered as a probable primary site. We present a case in which a testicular metastasis was the presenting sign and the only site of spread of prostatic carcinoma.

An 89-year-old male presented with a 36-hour history of right testicular pain and swelling. There was no dysuria. There was a mass at the lower pole of the right testis. Rectal examination revealed a smoothly enlarged firm right lobe of prostate. Ultrasound confirmed a $3 \mathrm{~cm}$ lesion at the lower pole of the testis. Alpha-fetoprotein and $b$ human chorionic gonadotrophin (HCG) were normal. Prostate-specific antigen (PSA) was $7.2 \mathrm{ng} / \mathrm{ml}$, but this was associated with a coliform urinary tract infection. A right radical orchidectomy was performed. Histology revealed an adenocarcinoma staining strongly positive for PSA and prostatic specific acid phosphatase (PSAP), confirming a diagnosis of metastatic prostate carcinoma. An isotope bone scan and computer tomography $(\mathrm{CT})$ revealed no metastases. 